Actualidades en Psicología, 31(122), 2017, 31-43

ISSN 2215-3535

http://revistas.ucr.ac.cr/index.php/actualidades

DOI: http://dx.doi.org/10.15517/ap.v31i122.23346

\title{
Relaciones de pareja homosexual y heterosexual: un estudio comparativo
}

\section{Couple Relationships Homosexual And Heterosexual: A Comparative Study}

\author{
Felipe E. García ${ }^{1}$ \\ Universidad Santo Tomás, Chile \\ Carlos García Escobar ${ }^{2}$ \\ Helly Hein ${ }^{3}$ \\ Álvaro Hernández ${ }^{4}$ \\ Patricio Torres ${ }^{5}$ \\ Rayén Valdebenito ${ }^{6}$ \\ Camilo Vera ${ }^{7}$ \\ Universidad de Concepción, Chile
}

Resumen. Esta investigación tiene el propósito de evaluar y comparar a personas heterosexuales y homosexuales, con relaciones de pareja estables, respecto a los componentes de la teoría triangular del amor, tácticas de resolución de conflictos, ajuste con la pareja y bienestar psicológico. Participaron 157 adultos jóvenes chilenos, 77 homosexuales (30 lesbianas y 47 gays) y 80 heterosexuales (45 mujeres y 35 hombres). Se utilizaron la Escala de Ajuste Diádico, la Escala Triangular del Amor de Sternberg, la Flourishing Scale de Diener y el Conflict Resolution Style Inventory de Kurdek. Los resultados muestran algunas diferencias significativas en tácticas de resolución de conflicto, componentes del amor y ajuste en la pareja, según la orientación sexual; además existen diferencias entre las mujeres que conviven y no conviven.

Palabras clave. Satisfacción marital, manejo de conflicto, amor, pareja heterosexual, pareja homosexual.

Abstract. The aim of this study was to evaluate and compare heterosexual and homosexual people who are in stable relationships, with respect to the components of the triangular theory of love: conflict resolution tactics, couple adjustment and psychological wellbeing. The participants were comosed of 157 young adults Chileans: 77 homosexuals (30 lesbians and 47 gay men) and 80 heterosexuals (45 women and 35 men). The instruments used were the Dyadic Adjustment Scale, the Sternberg's Triangular Love Scale, the Flourishing Scale of Diener and Conflict Resolution Style Inventory of Kurdek. The results show that there are significant differences in some conflict resolution tactics, components of love between partners, and couple adjustment according to sexual orientation. In addition, there are also differences between women living and not living with a partner.

Keywords. Marital satisfaction, conflict management, love, heterosexual couple, homosexual couple

\footnotetext{
${ }^{1}$ Felipe E. García. Universidad Santo Tomás, Chile. Dirección postal: Avda. Arturo Prat 855, Concepción. Código postal: 4061501, Chile. E-mail: felipegarciam@yahoo.es

${ }^{2}$ Carlos García Escobar. Universidad de Concepción, Chile. E-mail: carlogarcia@udec.cl

${ }^{3}$ Helly Hein. Universidad de Concepción, Chile. E-mail: hhein@udec.cl

${ }^{4}$ Álvaro Hernández. Universidad de Concepción, Chile. E-mail: alvhernandez@udec.cl

${ }^{5}$ Patricio Torres. Universidad de Concepción, Chile. E-mail: patorresm@udec.cl

${ }^{6}$ Rayén Valdebenito. Universidad de Concepción, Chile. E-mail: rayenvaldebenit@udec.cl

${ }^{7}$ Camilo Vera. Universidad de Concepción, Chile. E-mail: camilovera@udec.cl
}

\section{(c) $(1) \Theta \Theta$}




\section{Introducción}

La mayoría de los estudios que han abordado las relaciones de pareja como materia de interés se han centrado en las relaciones heterosexuales. Autores como Kurdek (1998; 2004), Peplau (1991) y Gottman et al. (2003) fueron pioneros en realizar estudios para entender a la pareja homosexual más allá de los prejuicios, considerando variables culturales y sociales.

Hoy en día, la investigación en el área está viviendo un proceso que busca derribar los antiguos prejuicios y estereotipos relacionados con esta materia, para abordarlo desde una perspectiva científica (Meccia, 2011). Estos estudios se han realizado principalmente en Europa y Norteamérica donde, si bien persisten acciones discriminatorias contra las personas homosexuales, han mostrado un cambio cultural que tiende a la inclusión y a la igualdad de derechos; ejemplo de ello es la legalización del matrimonio homosexual, hoy presente en varios países.

Contrario a estos cambios culturales, Chile ha mostrado, a través de su legislación, ser un país más bien conservador (Cornejo, 2011) y homofóbico (Barker \& Aguayo, 2012) donde el matrimonio homosexual no existe a pesar de que recientemente se concretó la posibilidad de un Acuerdo de Vida en Pareja, una suerte de contrato civil que regula las relaciones en personas que no están casadas, hetero u homosexuales, y que para algunas comunidades que agrupan a personas homosexuales no resulta satisfactorio pues está lejos de equipararse al matrimonio (Pérez, 2014).

Aun así, la sola discusión parlamentaria de esa ley ha incrementado la oposición política y religiosa a legalizar este tipo de unión sexual, una postura que ha permeado los discursos científicos sobre esta materia (Morán \& Vaggione, 2012), lo que se representa con algunas publicaciones que han abordado el tema desde una perspectiva contraria a los derechos de las minorías sexuales (Valenzuela, 2006; Zegers, Larraín \& Bustamante, 2007), homologando la homosexualidad a una enfermedad. Chile, además, presenta altas tasas de actitudes homofóbicas y discriminación por razones sexuales (Barrientos \& Cárdenas, 2013; Romero,
2011) en las que se cuentan, incluso, asesinatos (Hernández, 2013). Por eso, no es de extrañar la carencia de estudios científicos que exploren variables psicológicas y relacionales en la comunidad LGTB, por cuanto esta población se encuentra estigmatizada, es de difícil acceso o reticente a responder cuestionarios de naturaleza científica debido a la desconfianza que le genera.

El estudio del amor de pareja ha sido desarrollado, principalmente, por Sternberg (1989) quien caracteriza el amor como una emoción de tres componentes: compromiso, intimidad y pasión. El compromiso alude al interés y responsabilidad que se siente por una pareja y por la decisión de mantener la relación en el transcurso del tiempo. La intimidad alude al apoyo afectivo, la capacidad de compartir asuntos personales y profundos con su pareja, entre otros aspectos. La pasión hace referencia a la atracción física hacia la pareja. Los estudios de Sternberg (1989) muestran que la intimidad y el compromiso tienden a aumentar con el tiempo y profundizarse con la convivencia; en cambio, la pasión disminuye ante la progresiva reducción de incertidumbre y atención selectiva hacia la pareja.

Diversos estudios (Barrios \& Pinto, 2008; Maureira \& Maureira, 2012; Serrano \& Carreño, 1993) han evaluado estos componentes del amor, principalmente en jóvenes heterosexuales, en países como Bolivia, Chile y España; en todos ellos se encontró que la intimidad es el componente que alcanza más altos niveles, sin observar diferencias significativas según el sexo de los sujetos. Sin embargo, en estudiantes universitarios mexicanos, Mazadiego y Norberto (2011) encontraron que los hombres prefieren en sus relaciones mayor intimidad y pasión, dejando de lado el compromiso; mientas que las mujeres buscan relaciones con más compromiso y, en menor grado, pasión e intimidad.

En un estudio que incluyó heterosexuales de ambos sexos y gays, Albornoz (2009) encontró que no existen diferencias en los componentes del amor de acuerdo con su orientación sexual, aunque sí según el sexo, pues los hombres (heterosexuales y homosexuales) obtenían niveles más altos en compromiso dentro de 
sus relaciones, seguido de la intimidad y en último lugar la pasión. Las mujeres, en cambio, puntúan más alto en intimidad y, luego, el compromiso y la pasión. Este estudio no incluyó lesbianas debido a las dificultades para acceder a esta población; pero sí fueron incluidas en un estudio longitudinal llevado a cabo por Kurdek (1998), quien observó que las lesbianas mostraban mayores niveles de intimidad que los heterosexuales hombres y mujeres.

Otro factor importante que incide en las parejas es el modo en que resuelven sus conflictos, sobre todo si se considera el prejuicio de que las parejas homosexuales son más "inestables y violentas" que las heterosexuales (Sauré, 2014). Las parejas disfuncionales no se distinguen de las funcionales debido a la ausencia o presencia del conflicto ni por las discrepancias que existan entre sus miembros, sino más bien, por cómo resuelven estos desacuerdos (Gottman \& Silver, 2012). Es posible apreciar que la evidencia en torno a cómo las parejas homosexuales y heterosexuales se diferencian al momento de solucionar un conflicto no es concluyente; en algunos estudios no existen diferencias y, en otros, gays y lesbianas parecen tener relaciones menos conflictivas.

Por ejemplo, Metz, Rosser y Strapko (1994) muestran que los estilos de resolución de conflictos parecen ser similares entre heterosexuales y homosexuales. En otros estudios, las parejas gays parecen reproducir las dinámicas y roles tradicionales de las parejas heterosexuales, caracterizados por la dominancia de uno sobre el otro (Burin, Meller \& Birmele, 2000). Las relaciones lésbicas, por el contrario, representarían una relación simétrica entre dos personas, sin la dominancia característica de la pareja heterosexual y gay (Pisano, 2004).

Gottman et al. (2003) encontraron que, en comparación con las parejas heterosexuales, las parejas gay y lesbianas iniciaban y mantenían sus discusiones en un tono más positivo. Kurdek (2004) observó que las parejas homosexuales tienen menor tendencia a usar un estilo de resolución de conflictos en que uno de los individuos le demanda al otro que se retire o pierda y tienen mayor tendencia a sugerir posibles soluciones y compromisos. Montes (2009) explica estos últimos resultados señalando que las parejas gay tienden a pensar y reaccionar de forma similar, lo que los ayudaría a restablecer en ocasiones ciertas divergencias; a su vez, las parejas lésbicas buscan la comprensión mutua, optando por silenciar en vez de emitir un desacuerdo para propiciar la calma en el hogar, dejando de expresar aquellas cosas que les molesta o presienten como amenaza a su convivencia.

Tanto los componentes del amor como la táctica de resolución de conflicto (TRC) parecen tener incidencia en el ajuste de la pareja y en la satisfacción en su relación (Cassepp-Borges \& Martins, 2009). El ajuste en la pareja es un proceso de adaptación a la convivencia, siendo la satisfacción un componente del mismo, junto al consenso sobre asuntos importantes, la cohesión y la expresión de cariño (Spanier \& Cole, 1976).

Un estudio realizado en Estados Unidos con heterosexuales mostró que, en los hombres, el compromiso predice en mayor grado la satisfacción en la relación de pareja; mientras que, en las mujeres, la intimidad es el mejor predictor de ella (Lemieux \& Hale, 2000). En cuanto a parejas homosexuales, se ha observado un mayor nivel de satisfacción en parejas lesbianas que en parejas heterosexuales o gays (Kurdek, 1998; Metz et al., 1994; Zacks, Green \& Marrow, 1988). Kurdek (1998) sugiere que estos resultados podrían deberse a que las mujeres, indistintamente de su orientación sexual, son socializadas (en comparación a los hombres) hacia el cuidado y la expresividad hacia su pareja, resultando así en mayor satisfacción reportada.

Por otro lado, la TRC está también relacionada con la satisfacción de la pareja, siendo aquellas más satisfechas las que utilizan tácticas más positivas. Campos y Poulsen (2013), en un estudio con parejas heterosexuales chilenas, observaron que una resolución de conflictos orientada a la adaptación y aceptación del otro durante el noviazgo predecía un mayor ajuste y satisfacción en la pareja tras el matrimonio; observaron, además, que las personas casadas, al momento de enfrentarse a un conflicto, priorizan el mantenimiento del vínculo por sobre la confrontación. 
En relación al bienestar, los estudios sugieren que la satisfacción con la pareja es uno de los mayores predictores de bienestar subjetivo en las personas (Helgeson, 1994). Por ejemplo, en población chilena adulta se ha observado que aquellas personas que se encuentran en una relación de pareja presentaban un bienestar más alto que las personas solteras (Moyano \& Ramos, 2007; Vivaldi \& Barra, 2012).

Respecto a las diferencias por orientación sexual, anteriormente se consideraba a las personas homosexuales en comparación con los heterosexuales como infelices (ver Peplau \& Fingerhut, 2007). Sin embargo, los estudios muestran una gran similitud entre las parejas actuales en términos de estabilidad, felicidad y satisfacción (Kurdek, 1998). Araguez (2012), por ejemplo, encontró que no existe una diferencia significativa entre los niveles de bienestar psicológico en parejas heterosexuales y homosexuales argentinas en convivencia.

En síntesis, los datos reunidos sugieren que las parejas homosexuales y heterosexuales tienen niveles similares de amor, satisfacción con su relación y bienestar psicológico; las lesbianas obtienen niveles más altos en satisfacción de pareja en algunos estudios. También existirían diferencias en las TRC según la orientación sexual. Ante esto, surge la siguiente pregunta de investigación: ¿qué diferencias y similitudes existen en tipos del amor, estilos de resolución de conflictos, satisfacción de pareja y bienestar psicológico entre personas heterosexuales y homosexuales chilenas? El propósito del presente estudio es responder esta interrogante.

\section{Método}

\section{Diseño}

Se utilizó un diseño descriptivo y correlacional. Los datos se recogieron en una sola oportunidad, por lo que corresponde a una investigación transversal.

\section{Población y participantes}

La población correspondió a 157 adultos (47.8\% mujer y $52.2 \%$ hombre), entre 18 y 40 años $(M=24.23$; $D T=5.17)$, rango correspondiente a la adultez joven
(Papalia, 2001), que se encontraban en una relación de pareja estable de al menos un mes, pudiendo convivir o no, y que no tuvieran hijos. Homosexuales eran 77 (30 lesbianas y 47 gays) y heterosexuales 80 (45 mujeres y 35 hombres). Un 26.8\% $(n=42)$ de los participantes se encontraba conviviendo con su pareja y el $73.2 \%$ restante $(n=115)$ no convivía. La mayoría de ellos contaba con educación universitaria completa o incompleta $(84.3 \%$ ) y se definía como clase media $(87.3 \%)$. Previo a su participación en el estudio, se les solicitó firmar una carta de consentimiento informado que daba cuenta de la voluntariedad de la participación, el anonimato de su identidad, la libertad para abandonar el estudio cuando quisieran y el resguardo de los datos por parte de los investigadores, siguiendo las directrices éticas para la investigación en psicología normadas por la American Psychological Association (2010).

\section{Instrumentos}

Escala de ajuste diádico (Spanier \& Cole, 1976). Se utilizó para evaluar el ajuste con la pareja y la satisfacción marital. Consta de 32 ítems que se responden en una escala tipo Likert y la puntuación total oscila entre 0 y 151 puntos. Sus propiedades psicométricas en Chile fueron estudiadas por Tapia y Poulsen (2009); mostraron adecuados índices de validez (concurrente y de constructo) y confiabilidad. Para el presente estudio se eliminaron tres ítems cuyas respuestas exigían que los encuestados se encontraran conviviendo, lo que no era el caso de la mayoría de los participantes. Se obtuvo un $\alpha=.84$.

Escala triangular del amor (Sternberg, 1986). Usada para medir el amor y sus componentes, consta de 45 ítems, dividida en 3 subescalas: compromiso, pasión e intimidad, cada una con 15 ítems. Utiliza una escala tipo Likert desde 1 (no del todo) a 9 (extremadamente). Su adaptación para Chile la realizaron Maureira y Maureira (2012); obtuvieron adecuados índices de confiabilidad y validez de constructo. En el presente estudio se obtuvieron los siguientes índices de confiabilidad según $\alpha$ de Cronbach: intimidad .92, compromiso .94, pasión .91 y total .97. 
Conflict Resolution Style Inventory (Kurdek, 1994). Fue usada para evaluar las TRC en la pareja. Está compuesta por 16 ítems que se responden en una escala de Likert de cinco puntos desde 1 (nunca) a 5 (siempre). Se compone de cuatro subescalas: negociación, confrontación, retirada y sumisión. La escala fue traducida y adaptada por los autores del presente estudio, se realizó un análisis factorial exploratorio (AFE) que encontró los mismos cuatro factores y la consistencia interna obtenida para la escala total fue de $\alpha=.74$; negociación .64, confrontación .68, retirada .58 y sumisión .65 .

Flourishing Scale (Diener et. al., 2010). Esta escala fue utilizada para evaluar el bienestar psicológico. Está compuesta por 8 ítems que se responden en una escala Likert de 1 (muy en desacuerdo) a 7 (muy de acuerdo). Fue adaptada para Chile por Ramírez (2015), confirmando su unidimensionalidad y obteniendo adecuada consistencia interna. En el presente estudio se obtuvo un $\alpha=.86$.

Encuesta de antecedentes personales. Se elaboró una encuesta para la obtención de datos tales como sexo, edad, orientación sexual, situación socioeconómica, tiempo de duración de la relación y escolaridad.

\section{Procedimiento}

El instrumento Conflict Resolution Style Inventory, de Kurdek, fue sometido a una traducción cruzada al español realizada por dos expertos. Posteriormente, se llevó a cabo una aplicación piloto a diez personas, con la finalidad de evaluar la comprensión de los ítems traducidos que permitieron algunas adecuaciones en el lenguaje.

Para acceder a los participantes se contactó a dos organizaciones chilenas pro-diversidad sexual, el Movimiento de Integración y Liberación Homosexual (MOVILH) y la Fundación Iguales, además de concurrir a centros de diversión utilizados por la población gay. A partir de las personas contactadas por estos medios y otras personas conocidas de los investigadores, se siguió la metodología de la bola de nieve para ampliar la muestra. Para la población heterosexual se buscaron personas que cumplieran con los mismos criterios que en el grupo homosexual, intentando equilibrar en sexo, edad, nivel socioeducacional y comuna de residencia. Cabe señalar que diez mujeres, pertenecientes a un colectivo lesbofeminista, se negaron a responder la encuesta.

Las encuestas fueron aplicadas entre los meses de agosto y octubre del 2014. Si bien todos los participantes fueron contactados en forma personal, un $69 \%(n=108)$ respondió la encuesta vía digital.

\section{Análisis de datos}

En primera instancia, se realizó un AFE de la Conflict Resolution Style Inventory para evaluar su validez de constructo y proceder a los siguientes análisis. Luego se hizo un análisis descriptivo de todos los instrumentos. Se estableció la correlación entre las variables de estudio en personas heterosexuales $y$ homosexuales en forma independiente, para luego comparar si existían diferencias significativas entre las correlaciones entre ambos grupos realizando en primer lugar la transformación z de Fischer para los valores $r$ y luego la $q$ de Cohen para estimar el tamaño del efecto de esa diferencia, cuando esta era significativa.

A continuación se hicieron comparaciones entre los dos sexos y las dos orientaciones sexuales (homosexual y heterosexual) usando para ello la $t$ de Student para muestras independientes, utilizando la $d$ de Cohen para estimar el tamaño del efecto. Finalmente, se realizó una regresión lineal múltiple para establecer los predictores del bienestar psicológico y el ajuste con la pareja en cada grupo, utilizando el valor $R^{2}$ para estimar el tamaño del efecto.

Para la evaluación de los tamaños de efecto, se consideraron para la $q$ de Cohen y $r$ los siguientes valores: .10 es pequeña, .30 es moderada y .50 es grande. Para la $d$ de Cohen se consideró que valores inferiores a .20 indican un efecto pequeño, .50 moderada y .80 grande. Para el $R^{2}$ un .02 es pequeño, .13 es mediano y .26 es grande (Cohen, 1992).

\section{Resultados}

Se procede a realizar un AFE de la Conflict Resolution Style Inventory de Kurdek. Para establecer el número 
de factores se utiliza el análisis paralelo de Horn (O’Connor, 2000), el cual mostró la existencia de cuatro factores que explicaron un $39 \%$ de la varianza y cuyos ítems coincidieron con la propuesta original de Kurdek acerca del instrumento. Los ítems por factor se determinaron a través de la factorización de ejes principales con rotación Promax. Los resultados se observan en la tabla 1 .

Se calcularon los estadísticos descriptivos de las principales variables de estudio, tanto en el grupo total como en homosexuales y heterosexuales (ver tabla 2). Se puede observar que, en el grupo total, la media

Tabla 1

Factor (varianza explicada) y cargas factoriales de la Escala de Conflicto

\begin{tabular}{|c|c|c|c|c|}
\hline & \multicolumn{4}{|c|}{ Factor } \\
\hline & 1 & 2 & 3 & 4 \\
\hline \multicolumn{5}{|c|}{ Confrontación $(18.62 \%)$} \\
\hline ITEM 1 & .63 & & & \\
\hline ITEM 9 & .62 & & & \\
\hline ITEM 13 & .61 & & & \\
\hline ITEM 5 & .54 & & & \\
\hline \multicolumn{5}{|c|}{ Sumisión $(9.50 \%)$} \\
\hline ITEM 4 & & .32 & & \\
\hline ITEM 16 & & .73 & & \\
\hline ITEM 8 & & .61 & & \\
\hline ITEM 12 & & .55 & & \\
\hline \multicolumn{5}{|c|}{ Negociación (5.99\%) } \\
\hline ITEM 10 & & & .74 & \\
\hline ITEM 6 & & & .56 & \\
\hline ITEM 2 & & & .52 & \\
\hline ITEM 14 & & & .52 & \\
\hline \multicolumn{5}{|c|}{ Retirada (4.68\%) } \\
\hline ITEM 15 & & & & .68 \\
\hline ITEM 7 & & & & .50 \\
\hline ITEM 11 & & & & .46 \\
\hline ITEM 3 & & & & .41 \\
\hline
\end{tabular}

más alta en la escala de tipos de amor se obtuvo en intimidad $(M=115.3 ; D T=15.4)$. En cuanto a las TRC, el estilo de negociación $(M=15.1 ; D T=2.4)$ tiene una media más elevada que los otros tres estilos.

Se realiza una comparación por sexo en cada variable de estudio, sin encontrarse diferencias significativas entre hombre y mujer en ninguna variable. Luego se hace una comparación entre homosexuales y heterosexuales, encontrando solo diferencias significativas en la TRC de sumisión, t(139) = 3.374; $p>.001 ; d=.57$, los homosexuales obtuvieron puntajes más altos $(M=9.12 ; D T=3.08)$ que los heterosexuales $(M=7.53 ; D T=2.49)$.

Posteriormente, se calcularon las diferencias entre grupos con base en si se encontraban conviviendo o no. Entre hombres gay y heterosexuales no se encuentran diferencias significativas en ninguna de las variables entre quienes conviven y quienes no. En lesbianas, observamos que en pasión, compromiso y puntaje total en la escala de amor presentan puntajes más altos quienes se encuentran conviviendo con sus parejas que quienes no. En mujeres heterosexuales, el bienestar psicológico, compromiso, satisfacción y ajuste con la pareja son significativamente mayores en el grupo que convive que en el grupo que no convive (ver tabla 3).

Se evaluaron las relaciones entre variables mediante el coeficiente de correlación $\mathrm{r}$ de Pearson en forma separada en homosexuales (ver tabla 4) y en heterosexuales (ver tabla 5) a fin de comparar los resultados en ambos grupos. En homosexuales se observa el ajuste con la pareja correlaciona más alto con el tipo de amor de intimidad $(r=.53)$ y la TRC de confrontación $(r=-.43)$ y negociación $(r=.42)$. A su vez, el bienestar psicológico correlaciona más alto con el tipo de amor de compromiso $(r=.24)$ y la TRC de negociación $(r=.23)$. En heterosexuales, el ajuste con la pareja correlaciona más alto con el tipo de amor de intimidad $(r=.56)$ y la TRC de negociación $(r=.40)$. A su vez, con bienestar psicológico, las correlaciones más altas se obtuvieron con el amor total $(r=.33)$ y con la pasión $(r=.25)$, así como la TRC de confrontación $(r$ $=-.25)$. A su vez, se observa en ambos grupos que los 
Tabla 2

Estadísticos descriptivos de las variables de estudio

\begin{tabular}{lcccccccccccc}
\hline & \multicolumn{3}{c}{ Grupo total $(\mathrm{n}=157)$} & \multicolumn{3}{c}{ Homosexuales $(\mathrm{n}=77)$} & \multicolumn{3}{c}{ Heterosexuales $(\mathrm{n}=80)$} \\
\cline { 2 - 10 } Variables & M & DT & Mín & Máx & M & DT & Mín & Máx & M & DT & Mín & Máx \\
\hline Amor - Intimidad & 115.3 & 15.4 & 58 & 135 & 115.7 & 15.0 & 70 & 135 & 115.8 & 14.8 & 58 & 135 \\
Amor - Compromiso & 112.4 & 20.4 & 47 & 135 & 113.8 & 19.3 & 60 & 135 & 112.2 & 20.2 & 53 & 135 \\
Amor - Pasión & 103.6 & 19.9 & 48 & 135 & 105.9 & 20.7 & 48 & 135 & 102.1 & 18.7 & 58 & 133 \\
Amor total & 331.5 & 50.7 & 175 & 405 & 334.9 & 51.2 & 179 & 405 & 328.3 & 50.3 & 175 & 396 \\
TRC - Negociación & 15.1 & 2.4 & 4 & 20 & 15.4 & 2.0 & 10 & 20 & 14.7 & 2.7 & 4 & 19 \\
TRC - Confrontación & 8.9 & 2.8 & 4 & 17 & 9.2 & 3.1 & 4 & 17 & 8.7 & 2.6 & 4 & 16 \\
TRC - Retirada & 9.5 & 2.8 & 4 & 18 & 9.7 & 3.1 & 4 & 16 & 9.5 & 2.6 & 5 & 18 \\
TRC - Sumisión & 8.2 & 2.8 & 4 & 16 & 9.1 & 3.1 & 4 & 16 & 7.5 & 2.5 & 4 & 14 \\
Ajuste con la pareja & 101.0 & 12.3 & 56 & 125 & 117.5 & 15.4 & 82 & 144 & 115.9 & 12.1 & 86 & 139 \\
Bienestar Psicológico & 34.3 & 4.3 & 9 & 54 & 34.7 & 4.1 & 15 & 40 & 33.8 & 4.3 & 9 & 40 \\
\hline
\end{tabular}

TRC= Táctica de resolución de conflicto

Tabla 3

Medias, desviaciones estándar, valor $T y d$ de Cohen por tipo de relación, en mujeres lesbianas y heterosexuales

\begin{tabular}{|c|c|c|c|c|c|c|c|}
\hline \multirow[t]{2}{*}{ Variable } & \multirow[t]{2}{*}{ Tipo de relación } & \multicolumn{3}{|c|}{$\begin{array}{c}\text { Lesbianas } \\
\text { (convive= }=11 \text {; no convive }=18 \text { ) }\end{array}$} & \multicolumn{3}{|c|}{$\begin{array}{c}\text { Mujeres heterosexuales } \\
\text { (conviven=9; no conviven=31) }\end{array}$} \\
\hline & & $M$ & $D E$ & & $M$ & $D E$ & \\
\hline \multirow[t]{2}{*}{ Amor-Pasión } & Convive & 117.64 & 14.68 & $\mathrm{t}=2.517^{*}$ & 108.22 & 13.65 & $\mathrm{t}=1.552$ \\
\hline & No convive & 98.33 & 22.60 & $\mathrm{~d}=1.01$ & 98.79 & 16.52 & $\mathrm{~d}=0.62$ \\
\hline \multirow[t]{2}{*}{ Amor - Compromiso } & Convive & 124.91 & $15 ., 92$ & $\mathrm{t}=2.070^{*}$ & 121.13 & 4.79 & $\mathrm{t}=2.701 * *$ \\
\hline & No convive & 109.88 & 22.46 & $\mathrm{~d}=0.77$ & 110.10 & 20.68 & $\mathrm{~d}=0.74$ \\
\hline \multirow[t]{2}{*}{ Amor - Intimidad } & Convive & 122.64 & 15.04 & $\mathrm{t}=1.393$ & 123.00 & 8.14 & $\mathrm{t}=1.437$ \\
\hline & No convive & 113.94 & 16.78 & $\mathrm{~d}=0.55$ & 114.13 & 17.86 & $\mathrm{~d}=0.64$ \\
\hline \multirow[t]{2}{*}{ Amor-Total } & Convive & 365.18 & 43.36 & $\mathrm{t}=2.058^{*}$ & 349.38 & 20.01 & $\mathrm{t}=1.531$ \\
\hline & No convive & 323.82 & 56.63 & $\mathrm{~d}=0.82$ & 320.41 & 51.99 & $\mathrm{~d}=0.74$ \\
\hline \multirow[t]{2}{*}{ TRC-Negociación } & Convive & 15.91 & 1.92 & $\mathrm{t}=0.552$ & 16.11 & 1.83 & $\mathrm{t}=1.895$ \\
\hline & No convive & 15.50 & 1.95 & $\mathrm{~d}=0.21$ & 14.10 & 3.02 & $\mathrm{~d}=0.80$ \\
\hline \multirow[t]{2}{*}{ TRC-Confrontación } & Convive & 8.45 & 3.42 & $\mathrm{t}=-0.145$ & 8.89 & 2.62 & $\mathrm{t}=-0.250$ \\
\hline & No convive & 8.61 & 2.40 & $\mathrm{~d}=-0.05$ & 9.17 & 3.00 & $\mathrm{~d}=-0.10$ \\
\hline \multirow[t]{2}{*}{ TRC-Retirada } & Convive & 10.27 & 3.23 & $\mathrm{t}=1.181$ & 8.33 & 1.58 & $\mathrm{t}=-1.413$ \\
\hline & No convive & 8.94 & 2.75 & $\mathrm{~d}=0.44$ & 9.48 & 2.28 & $\mathrm{~d}=-0.59$ \\
\hline \multirow[t]{2}{*}{ TRC-Sumisión } & Convive & 8.27 & 3.00 & $\mathrm{t}=-0.782$ & 7.56 & 2.30 & $\mathrm{t}=0.250$ \\
\hline & No convive & 9.17 & 2.98 & $\mathrm{~d}=-0.30$ & 7.32 & 2.51 & $\mathrm{~d}=0.10$ \\
\hline \multirow[t]{2}{*}{ Ajuste con la pareja } & Convive & 124.09 & 17.31 & $\mathrm{t}=0.821$ & 121.38 & 5.04 & $\mathrm{t}=2.749 *$ \\
\hline & No convive & 118.93 & 14.18 & $\mathrm{~d}=0.33$ & 111.65 & 13.67 & $\mathrm{~d}=0.95$ \\
\hline \multirow[t]{2}{*}{ Bienestar psicológico } & Convive & 34.55 & 3.17 & $\mathrm{t}=-1.212$ & 36.44 & 2.40 & $\mathrm{t}=2.285^{*}$ \\
\hline & No convive & 35.94 & 2.92 & $\mathrm{~d}=-0.46$ & 32.35 & 5.17 & $\mathrm{~d}=1.01$ \\
\hline
\end{tabular}

TRC $=$ Táctica de resolución de conflicto. ${ }^{*} p<.05 ; * * p<.01 ; * * * p<.001$. 
tipos de amor y TRC correlacionan más alto con el ajuste con la pareja que con el bienestar psicológico. La correlación entre ajuste con la pareja y el bienestar psicológico es no significativa en homosexuales $(r=$ .17) y significativa pero baja en heterosexuales $(r=.28)$.

Se compararon las correlaciones entre heterosexuales y homosexuales; la diferencia más importante estaba en la relación pasión-negociación (homosexuales: $r=$ .18; heterosexuales: $r=.39$ ), pero esta diferencia no resultó significativa $(z=-1.33 ; q$ de Cohen $=-.23)$. Se calcularon también los predictores del ajuste con la pareja utilizando la regresión lineal múltiple (RLM), con los tipos de amor y TRC como predictores. Las regresiones se hicieron por cada orientación sexual y luego por el total de la muestra. En los análisis no se observaron predictores significativos de ajuste con la pareja en hombres gays y heterosexuales y mujeres heterosexuales. En el caso de las lesbianas, el modelo resulta significativo $\left(F_{(7.16)}=6.687 ; p<.001\right)$,

Tabla 4

Correlaciones de Pearson entre las variables de estudio en gays y lesbianas $(n=77)$

\begin{tabular}{|c|c|c|c|c|c|c|c|c|c|}
\hline Variable & 2 & 3 & 4 & 5 & 6 & 7 & 8 & 9 & 10 \\
\hline 1. Amor-Pasión & $.88^{* * *}$ & $.75^{* * *}$ & $.94 * * *$ & .18 & .02 & .06 & .09 & $.38 * *$ & .18 \\
\hline 2. Amor - Compromiso & - & $.86^{* * *}$ & $.97 * * *$ & .23 & -.09 & -.04 & -.09 & $.47^{* * *}$ & $.24 *$ \\
\hline 3. Amor - Intimidad & & - & $.91 * * *$ & $.33^{* *}$ & -.17 & -.09 & -.08 & $.53 * * *$ & .15 \\
\hline 4. Amor - Total & & & - & .23 & -.07 & -.02 & -.03 & $.47^{* * *}$ & .19 \\
\hline 5. TRC - Negociación & & & & - & $-.29 *$ & -.16 & $-.36 * *$ & $.42 * * *$ & .23 \\
\hline 6. TRC - Confrontación & & & & & - & .21 & $.49 * * *$ & $-.43 * * *$ & .14 \\
\hline 7. TRC - Retirada & & & & & & - & .02 & -.09 & -.08 \\
\hline 8. TRC - Sumisión & & & & & & & - & $-.27^{*}$ & .01 \\
\hline 9. Ajuste con la pareja & & & & & & & & - & .17 \\
\hline 10. Bienestarpsicológico & & & & & & & & & - \\
\hline
\end{tabular}

Tabla 5

Correlaciones de Pearson entre las variables de estudio en hombres y mujeres heterosexuales $(n=80)$

\begin{tabular}{|c|c|c|c|c|c|c|c|c|c|}
\hline Variable & 2 & 3 & 4 & 5 & 6 & 7 & 8 & 9 & 10 \\
\hline 1. Amor-Pasión & $.87 * * *$ & $.70^{* * *}$ & $.93^{* * *}$ & $.39 * * *$ & -.13 & -.08 & .03 & $.48^{* * *}$ & $.25^{*}$ \\
\hline 2. Amor - Compromiso & - & $.83^{* * *}$ & $.97 * * *$ & $.40 * * *$ & $-.25^{*}$ & -.17 & -.01 & $.47 * * *$ & .20 \\
\hline 3. Amor - Intimidad & & - & $.89 * * *$ & $.34 * *$ & $-.36^{* *}$ & $-.25^{*}$ & -.06 & $.56^{* * *}$ & .16 \\
\hline 4. Amor - Total & & & - & $.40^{* * *}$ & $-.27 *$ & -.16 & .00 & $.53^{* * *}$ & $.33^{* *}$ \\
\hline 5. TRC - Negociación & & & & - & -.18 & $-.31 * *$ & -.12 & $.40^{* *}$ & .21 \\
\hline 6. TRC - Confrontación & & & & & - & $.41 * * *$ & .11 & -.24 & $-.25^{*}$ \\
\hline 7. TRC - Retirada & & & & & & - & $.33^{* *}$ & $-.38 * *$ & -.05 \\
\hline 8. TRC - Sumisión & & & & & & & - & .02 & -.01 \\
\hline 9. Ajuste con la pareja & & & & & & & & - & $.28 *$ \\
\hline 10. Bienestar psicológico & & & & & & & & & - \\
\hline
\end{tabular}

TRC: Táctica de resolución de conflictos. ${ }^{*} p<.05 ;{ }^{* *} p<.01 ; * * * p<.001$ 
Tabla 6

Puntaje beta (coeficiente estandarizado), t de studenty $\mathrm{R}^{2}$ en regresión lineal múltiple de variables predictoras sobre ajuste en la pareja y bienestar psicológico.

\begin{tabular}{|c|c|c|c|c|c|c|}
\hline \multirow[b]{3}{*}{ Variable predictora } & \multicolumn{2}{|c|}{ Ajuste en la pareja $\left(\mathrm{R}^{2}=.75\right)$} & \multicolumn{2}{|c|}{ Ajuste en la pareja $\left(\mathrm{R}^{2}=.35\right)$} & \multicolumn{2}{|c|}{ Bienestar psicológico $\left(\mathrm{R}^{2}=.13\right.$} \\
\hline & \multicolumn{2}{|c|}{ Lesbianas $(n=24)$} & \multicolumn{2}{|c|}{ Todos/as $(\mathrm{n}=107)$} & \multicolumn{2}{|c|}{ Todos/as $(n=134)$} \\
\hline & Beta & $t$ & Beta & $t$ & Beta & $T$ \\
\hline Amor - Pasión & .01 & .028 & .18 & 1,137 & -.24 & $-1,354$ \\
\hline Amor - Intimidad & .15 & .601 & .35 & $1.988^{*}$ & -.07 & -.427 \\
\hline Amor- Compromiso & .17 & .549 & -.09 & -.439 & .52 & $2.31 *$ \\
\hline TRC - Negociación & .18 & 1.263 & .19 & $1.997 *$ & .13 & 1.375 \\
\hline TRC-Confrontación & -.53 & $-2.812 * *$ & -.18 & -1.878 & .11 & 1.146 \\
\hline TRC - Retirada & .07 & .397 & 0 & .007 & -.13 & -1.415 \\
\hline TRC - Sumisión & -.26 & -1.779 & -.04 & -.417 & .02 & .247 \\
\hline
\end{tabular}

TRC= Táctica de resolución de conflicto; $*: p<.05 ; * *: p<.01$.

con un $R^{2}$ de $.75\left(R^{2}\right.$ adj $\left.=.64\right)$, siendo las tácticas de confrontación y de sumisión los únicos predictores significativos, en este caso negativos.

En el grupo total, se obtuvo también un modelo significativo $\left(F_{(7.99)}=7.742 ; p<.001\right.$, con un $R^{2}$ de $.35\left(R^{2}\right.$ adj $\left.=.31\right)$. Los predictores significativos de ajuste con la pareja fueron la intimidad y la táctica de negociación, ambos positivos.

Secalcularon los predictores del bienestarpsicológico utilizando la RLM con el método introducir, con los tipos de amor y TRC como predictores. Las regresiones se hicieron por cada orientación sexual y luego por el total de la muestra. En los análisis no se observaron predictores significativos de bienestar psicológico en ninguna orientación sexual. En el grupo total, se obtuvo un modelo significativo $\left(F_{(7.126)}=2.762 ; p<\right.$ $.05)$, con un $R^{2}$ de $.13\left(\mathrm{R}^{2}\right.$ adj=.09). El único predictor positivo significativo de bienestar psicológico fue el compromiso (ver tabla 6 para los modelos que resultaron significativos).

\section{Discusión}

Uno de los propósitos de este estudio fue establecer y comparar las TRC de pareja en personas homosexuales y heterosexuales. Ante la carencia de instrumentos validados para evaluar esta variable en población chilena, se tradujo y adaptó una escala elaborada por
Kurdek (1994). Esta escala mostró ser fiable y válida para la población con la que se trabajó; sin embargo, si bien el AFE muestra la misma configuración de factores de la escala original, la varianza explicada por dichos factores es baja, por lo que se requiere seguir estudiando esta escala en el futuro. De todos modos, los resultados fueron considerados satisfactorios para continuar con los siguientes análisis.

En la observación de los estadísticos descriptivos de las escala triangular del amor, se constató que la intimidad alcanza los puntajes más altos, seguida del compromiso y luego la pasión, lo que coincide con otros estudios (Barrios \& Pinto, 2008; Maureira \& Maureira, 2012; Serrano \& Carreño, 1993). Asimismo, la TRC más utilizada por los participantes fue la negociación, una táctica considerada positiva por Kurdek (1998).

La ausencia de diferencias por sexo (hombre y mujer) con independencia de su orientación sexual, en todas las variables medidas, también coincide con varios estudios que señalan que los componentes del amor, TRC, ajuste con la pareja y bienestar psicológico son equivalentes entre los sexos (García, Wlodarczyk, Reyes, San Cristóbal \& Solar, 2014; Metz et al., 1994).

En el caso de la orientación sexual, se observa una ausencia de diferencias entre los grupos en los componentes del amor y en el bienestar psicológico. 
Estos hallazgos muestran que, efectivamente, las parejas homosexuales no tienen diferencias en sus niveles de bienestar individual en relación con las parejas heterosexuales (Araguez, 2012). En cuanto a las TRC, los homosexuales muestran mayor sumisión que heterosexuales; en otras palabras, gays y lesbianas "ceden" más ante las exigencias de su pareja en situaciones de conflicto, una suerte de evitación que preserva el clima de armonía, observación que ya había sido mencionada por Castañeda (1999). Metz et al. (1994) sugieren que las parejas del mismo sexo darían mayor prioridad a hacer esfuerzos por mantener un clima positivo en sus relaciones porque no poseen los mismos soportes sociales que los heterosexuales, que cuentan con reconocimiento legal, visibilidad y apoyo social, aunque eso signifique ceder con mayor frecuencia ante las demandas de su pareja.

La comparación en las variables entre quienes conviven o no, para cada orientación sexual, también mostró resultados interesantes. Por ejemplo, se observó inexistencia de diferencias en hombres, ya sean gays o heterosexuales, con lo que se muestra que la convivencia no modifica la relación en ninguna de las variables medidas. Distinto ocurre con las lesbianas, en las que la convivencia parece mejorar muchos aspectos vinculados al amor, como el compromiso y la intimidad, que pueden desarrollarse en mejor medida. En mujeres heterosexuales, se observa también un mejoramiento de la calidad de la relación, aumentando el compromiso, el ajuste en la relación y el bienestar psicológico.

$\mathrm{Al}$ momento de establecer predictores del ajuste en la pareja, solo en las lesbianas aparecen predictores significativos de un menor ajuste; en este caso, la confrontación y la sumisión. Resulta relevante considerar que la sumisión predice menor ajuste en las lesbianas y a su vez, como ya se señaló, ellas tienden más a la sumisión que las mujeres heterosexuales. Como ya se plantó, la sumisión posibilita evitar la escalada de conflicto para conservar la armonía, pero este resultado puede dar la razón a Castañeda (1999), quien indica que evitar la expresión permite mantener una paz casi absoluta, pero falsa.
Sin considerar la orientación sexual, se observó también que en el grupo total de participantes, resultan predictores positivos del ajuste en la pareja la intimidad y la negociación, lo que coincide también con otros estudios (Pérez \& Estrada; 2006; Ottazzi, 2009; García, Fuentes \& Sánchez, 2016). Según García et al. (2016), la relevancia de la intimidad como predictor del ajuste demuestra que los niveles de confianza y la vinculación emocional sólida, en las relaciones de pareja establecidas en la adultez temprana, favorecen una percepción positiva de la relación, aumentando los niveles de satisfacción. Dato similar fue encontrado por Carillo (2004), quien señaló que cuando las personas perciben que su pareja utiliza estilos de comunicación positivos, cualidad propia tanto de la intimidad como de la negociación, presentan mayor satisfacción con su relación

Al evaluar el bienestar psicológico, no se encontraron predictores significativos al considerar las distintas orientaciones sexuales, pero sí al considerar al grupo total. En este caso, el único predictor significativo del bienestar psicológico fue el compromiso, tal como se ha encontrado en otros estudios (Cajiao, Morales, Garzón, Benavides \& Acevedo, 2013; Meza, 2011; Peplau \& Fingernhut, 2007). Al parecer, estar en una relación estable y que se proyecta en el futuro, fortalece la satisfacción personal, brindando seguridad y mirada de futuro. Nina (2011) revela en su estudio que el compromiso es un elemento significativo para las parejas al facilitar en las personas un sentido de pertenencia e identidad

Este estudio presenta algunas limitaciones a considerar. En primer lugar, su diseño transversal y no experimental impide establecer relaciones de causa y efecto. Si bien se establecen algunas variables que pueden predecir otras, el diseño es correlacional por lo que la consignación de una variable como predictora es más teórica que empírica. Realizar estudios longitudinales que permitan evaluar el ajuste en la pareja, el bienestar y sus predictores a lo largo del tiempo, podría responder mejor esta interrogante.

Además, el acceso a la muestra no fue aleatoria, algo imposible considerando su naturaleza, lo que impide 
generalizar los resultados ante la posibilidad de haber caído en algún sesgo de selección. Ejemplo de lo anterior, es que 10 lesbianas se negaron a responder la encuesta al considerar que este estudio estaba realizado desde la heteronorma. Estas mujeres eran militantes de un movimiento lesbofeminista que se autodenomina como radical, un enfoque caracterizado por considerar a la heterosexualidad como una institución opresiva de poder (Hernández, 2013). El grupo de lesbianas evaluadas, por lo tanto, podría no representar al conjunto de lesbianas existentes en la población. Se sugiere que, para acceder a las dinámicas relacionales en el grupo más radical de lesbianas, se aborde desde un estudio más bien cualitativo.

Una última limitación, no menos importante, es la ausencia de pruebas de invarianza factorial de los instrumentos utilizados; si bien estos han sido validados para su uso en el contexto chileno, los resultados encontrados podrían explicarse en el diferente comportamiento psicométrico de los instrumentos entre los grupos y no a diferencias reales en el constructo evaluado. Por tal motivo, la realización de futuros estudios con poblaciones más grandes utilizando estos instrumentos permitiría la realización de pruebas de invarianza y, de ese modo, superar esta dificultad.

Se espera que estudios posteriores incorporen variables que no fueron medidas en la investigación, tales como los roles sexuales (Barra, 2010), la participación de la población homosexual en comunidades LGBT y las redes sociales de apoyo percibidas (Blair \& Holmberg, 2008). Igualmente, en consideración de los cambios legales que ha experimentado el país (como el Acuerdo de Vida en Pareja), se podrían realizar estudios para explorar el impacto que estas leyes tienen en la satisfacción y bienestar global de las personas homosexuales en Chile.

\section{Referencias}

Albornoz, V. (2009). Relación entre los componentes del amor de la teoría de Sternberg y la felicidad en heterosexuales y hombres homosexuales en una relación de pareja. (Tesis de licenciatura). Universidad de Talca, Chile.

American Psychological Association (2010). Ethical principles of psychologists and code of conduct. Washington DC, USA: APA.
Araguez, M. (2012). Parejas homosexuales y heterosexuales en concubinato: Bienestar psicológico y orientación del rol sexual. (Tesis de licenciatura). Universidad Abierta Interamericana, Buenos Aires, Argentina.

Barker, G. \& Aguayo, F. (2012). Masculinidades y políticas de equidad de género: Reflexiones a partir de la Encuesta IMAGES y una revisión de politicas en Brasil, Chile y México. Rio de Janeiro: Promundo.

Barra, E. (2010). Bienestar psicológico y orientación de rol sexual en estudiantes universitarios. Terapia Psicológica, 28(1), 119-125. doi: 10.4067/S071848082010000100011

Barrientos,J.\&Cárdenas,M.(2013).Homofobiaycalidad de vida de gay y lesbianas: Una mirada psicosocial. Psykhe, 22(1), 3-14. doi: 10.7764/psykhe.22.1.553

Barrios, A. \& Pinto, B. (2008). El concepto de amor en la pareja. Ajayu, 6(2), 144-164.

Blair, K. \& Holmberg, D. (2008). Perceived social network support and well-being in same-sex versus mixed-sex romantic relationships. Journal of Social and Personal Relationships, 25(5), 769-791. doi: 10.1177/0265407508096695

Burin, M., Meller, I., \& Birmele, R. (2000). Varones: género y subjetividad masculina. Buenos Aires: Paidós.

Cajiao, G., Morales, D., Garzón, G., Benavides, L., \& Acevedo, J. (2013). Revisión de algunos avances en la literatura sobre variables predictoras concernientes al 'bienestar subjetivo'. Cuadernos Hispanoamericanos de Psicología, 13(1), 57-62.

Campos, S. \& Poulsen, G. (2013). Estrategias de resolución de conflicto en parejas con hasta 7 años de matrimonio, hijos y alto ajuste marital. Un estudio descriptivo relacional. Revista de Familias y Terapias, 22(34), 63-84.

Carrillo, L. (2004). Relación entre estilos de comunicación, manejo de conflicto y satisfacción en la relación de pareja. (Tesis de licenciatura). Universidad de las Américas, Puebla, México.

Cassepp-Borges, V. \& Martins, M. (2009). Versión Reducida de la Escala Triangular del Amor: 
Características del Sentimiento en Brasil. Revista Interamericana de Psicología, 43(1), 30-38.

Castañeda, M. (1999). La Experiencia Homosexual. Ciudad de México: Paidós.

Cohen, J. (1992). A power primer. Psychological Bulletin, 112(1), 155-159. doi: 10.1037/00332909.112.1.155

Cornejo, J. (2011). Configuración de la homosexualidad medicalizada en Chile. Sexualidad, Salud y Sociedad, O(9), 109-136.

Diener, E., Wirtz, D., Tov, W., Kim-Prieto, C., Choi, D., Oishi, S., \& Biswas-Diener, R. (2010). New wellbeing measures: Short scales to assess flourishing and positive and negative feelings. Social Indicator Research. 97(2), 143-156. doi: 10.1007/s11205-009-9493-y

García, F., Fuentes, R., \& Sánchez, A. (2016). Amor, satisfacción en la pareja y resolución de conflictos en adultos jóvenes. Ajayu, 14(2), 288-307.

García, F., Wlodarczyk, A., Reyes, A., San Cristóbal, C. C., \& Solar, C. (2014). Violencia en la pareja, apoyo social y bienestar psicológico en adultos jóvenes. Ajayu, 12(2), 246-265.

Gottman, J., Levenson, R., Swanson, C., Swanson, K., Tyson, R., \& Yoshimoto, D. (2003). Observing gay, lesbian, and heterosexual couples' relationships: Mathematical modeling of conflicts interaction. Journal of Homosexuality, 45(1), 65-91. doi: 10.1300/ J082v45n01_04

Gottman, J. \& Silver, N. (2012). Siete reglas de oro para vivir en pareja. Barcelona: Random House Mondadori.

Helgeson, V. (1994). Relation of agency and communion to well-being: Evidence and potential explanations. Psychological Bulletin, 116(3), 412-428. doi: 10.1037/0033-2909.116.3.412

Hernández, I. (2013). Daniel Zamudio: Hacia la construcción de una noción de ciudadanía pluralista radical. ISEES, 12, 143-156.

Kurdek, L. (1994). Conflict resolution styles in gay, lesbian, heterosexual nonparent, and heterosexual parent couples. Journal of Marriage and the Family, 56(3), 705-722. doi: $10.2307 / 352880$

Kurdek, L. (1998). Relationship outcomes and their predictors: Longitudinal evidence from heterosexual married, gay cohabiting, and lesbian cohabiting couples. Journal of Marriage and the Family, 60(3), 553568. doi; $10.2307 / 353528$

Kurdek, L. (2004). Are gay and lesbian cohabiting couples really different from heterosexual married couples? Journal of Marriage and the Family, 66(4), 880-900.

Lemieux, R. \& Hale, J. (2000). Intimacy, passion, and commitment among married individuals: Further testing of the triangular theory of love. Psychological Reports, 87(3), 941-948. doi: 10.2466/ pr0.2000.87.3.941

Maureira, F. \& Maureira, Y. (2012). Características de los componentes del amor de pareja en una muestra de estudiantes chilenos. Revista Irtacala, 15(2), 206-217.

Mazadiego, T. \& Norberto, J. (2011). El amor medido por la Escala Triangular de Sternberg. Psicolatina. 22, 1-10.

Meccia, E. (2011). La sociedad de los espejos rotos. Apuntes para una sociología de la gaycidad. Sexualidad, Saludy Sociedad, O(8), 131-148.

Metz, M., Rosser, B., \& Strapko, N. (1994). Differences in conflict-resolution styles among heterosexual, gay, and lesbian couples. Journal of Sex Research. 31(4), 293308. doi: 10.1080/00224499409551764

Meza, S. (2011). Bienestar psicológico y modelo de inversión en la relación de pareja en estudiantes universitarios de Lima Metropolitana. (Tesis de licenciatura). Pontificia Universidad Católica del Perú, Lima, Perú.

Montes, B. (2009). Patrones de comunicación, diferenciación y satisfacción en la relación de pareja: Validación y análisis de estas escalas en muestras españolas. Anales de Psicología, 25(2), 288-298.

Morán, J. \& Vaggione, J. (2012). Ciencia y religión (hétero)sexuadas: El discurso científico del activismo católico conservador sobre la sexualidad en Argentina y Chile. Contemporánea, 2(1), 159-185. 
Moyano, E. \& Ramos, N. (2007). Bienestar subjetivo: Midiendo satisfacción vital, felicidad y salud en población chilena de la Región Maule. Universum, 2(22), 177-193.

Nina, R. (2011). ¿Quénos mantiene juntos? Explorando el compromiso y las estrategias de mantenimiento en la relación marital. Revista Intercontinental de Psicología y Educación, 13(2), 197-220.

O'connor, B. P. (2000). SPSS and SAS programs for determining the number of components using parallel analysis and Velicer's MAP test. Behavior Research Methods, Instruments, \& Computers, 32(3), 396402. doi: 10.3758/BF03200807

Ottazzi, A. (2009). Estilos de amor, satisfacción y compromiso en relaciones de pareja estables. (Tesis de licenciatura). Pontificia Universidad Católica del Perú.

Papalia, D. (2001). Desarrollo Humano. Bogotá, Colombia: Mac Graw Hill.

Peplau, L. (1991). Lesbian and gay relationships. In J. Gongsiorek and J. Weinrich (Eds.), Homosexuality: Research implications for public policy (p. 177-196). Newbury Park, CA: Sage Publications.

Peplau, L. \& Fingerhut, A. (2007). The Close Relationships of Lesbians and Gay Men. Annual Review of Psychology, 58, 405-424. doi: 10.1146/ annurev.psych.58.110405.085701

Pérez, A. (2014, 25 de abril). Ni lo uno ni lo otro, sino todo lo contrario. ¿Unión civil o matrimonio gay? Rebelión, Chile. Recuperado de: www.rebelion.org/ noticia.php?id $=183790$

Pérez, G. \& Estrada, S. (2006). Intimidad y comunicación en cuatro etapas de la vida de pareja: Su relación con la satisfacción marital. Archivos Hispanoamericanos de Sexología, 12(2), 133-163.

Pisano, M. (2004). El triunfo de la masculinidad. Santiago de Chile: Surada.

Ramírez, P. (2015). Evaluación del modelo del estado completo de salud mental en una muestra de estudiantes chilenos. (Tesis doctoral). Universidad de Concepción, Chile.

Romero, D. (2011). Homosexualidad y familia: ¿integración o rechazo? (Tesis de licenciatura). Universidad Academia de Humanismo Cristiano, Santiago, Chile.

Sauré, G. (2014, 04 de agosto). Jacqueline van Rysselberghe: Parejas homosexuales son más inestables y violentas. La Nación, Chile. Recuperado de: http:// www.lanacion.cl/noticias/pais/politica/jacquelinevan-rysselberghe-parejas-homosexuales-son-masinestables-y-violentas/2014-08-04/100938.html

Serrano, G. \& Carreño, M. (1993). La teoría de Sternberg sobre el amor. Análisis empírico. Psicothema, 5(1), 151-167.

Spanier, G. \& Cole, C. (1976). Toward clarification and investigation of marital adjustment. International Journal of Sociology of the Family, 6(1), 121-146.

Sternberg, R. (1986). A triangular theory of love. Psychological Review, 93, 119-135.

Sternberg, R. (1989). El Triángulo del Amor. Intimidad, Amor, Compromiso. Buenos Aires: Paidos.

Tapia, L. \& Poulsen, G. (2009). La evaluación del ajuste marital. Santiago: Universidad del Desarrollo.

Valenzuela, C. (2006). La homosexualidad ¿es una patología? Respuesta desde la biología evolutiva. Revista de Psiquiatría Clínica, 43(2), 27-38.

Vivaldi, F. \& Barra, E. (2012). Bienestar psicológico, apoyo social percibido y percepción de salud en adultos mayores. Terapia Psicológica, 30(2), 23-29. doi: S0718-48082012000200002

Zacks, E., Green, R., \& Marrow, J. (1988). Comparing lesbian and heterosexual couples on the circumplex model: An initial investigation. Family Process, 27(4), 471-484. doi: 10.1111/j.1545-5300.1988.00471.x

Zegers, B., Larraín, M. \& Bustamante, F. (2007). Sobre la homosexualidad. Santiago de Chile: Mediterráneo.

Recibido: 7 de abril de 2016

Aceptado: 23 de marzo de 2017 
